\title{
Analysis of coagulation parameters in patients with COVID-19 in Shanghai, China
}

\author{
Ying Zou ${ }^{1,}$, Hongying Guo ${ }^{1, \S}$, Yuyi Zhang ${ }^{1}$, Zhengguo Zhang ${ }^{1}$, Yu Liu ${ }^{1}$, Jiefei Wang ${ }^{1}$, \\ Hongzhou Lu ${ }^{2, *}$, Zhiping Qian ${ }^{1, *}$
}

${ }^{1}$ Department of Severe hepatopathy, Shanghai Public Health Clinical Center, Fudan University, Shanghai, China;

${ }^{2}$ Department of Infectious Disease and Immunology, Shanghai Public Health Clinical Center, Fudan University, Shanghai, China.

SUMMARY To investigate the characteristic of coagulation function in 303 patients with Coronavirus disease 2019 (COVID-19), we evaluated the correlation between coagulation function and disease status. We retrospectively analyzed 303 patients diagnosed with COVID-19 and evaluated the clinical data of 240 patients who were discharged. The coagulation function of the two groups (mild and severe) was compared. Compared with the mild group, majority of patients in the severe group were male (76.9\% vs. $49.8 \%$ ) and elderly (median age 65 vs. 50), and the proportion with chronic underlying diseases was higher $(73.1 \%$ vs. $36.1 \%)$. There were 209 abnormalities $(69.0 \%)$ of coagulation parameters in 303 patients admitted to hospital. Comparison of various indexes of coagulation function between the two groups in admission, the proportion of abnormal coagulation indicators in the severe group was higher than that in the mild group $(100 \%$ vs. $66.1 \%)$. The median coagulation parameters in the severe group were higher than those in the mild group: international normalized ratio (1.04 vs. 1.01), prothrombin time (13.8 vs. 13.4) seconds, activated partial thromboplastin time (43.2 vs. 39.2) seconds, fibrinogen $(4.74 v s .4 .33) \mathrm{g} / \mathrm{L}$, fibrinogen degradation products $(2.61 v s .0 .99) \mu \mathrm{g} / \mathrm{mL}$, and D-dimer $(1.04$ vs. 0.43$) \mu \mathrm{g} / \mathrm{mL}$, the differences were statistically significant $(p<0.05)$. Coagulation dysfunction is common in patients with COVID-19, especially fibrinogen and D-dimer elevation, and the degree of elevation is related to the severity of the disease. As the disease recovers, fibrinogen and activated partial thromboplastin time also return to normal.

Keywords COVID-19, SARS-CoV-2, coagulation parameter, fibrinogen, D-dimer

\section{Introduction}

Coronavirus disease 2019 is caused by a novel beta coronavirus, on February 12, 2020, the International Committee on Taxonomy of Viruses (ICTV) named the virus SARS-CoV-2 (severe acute respiratory syndrome coronavirus 2), and on the same day, the World Health Organization (WHO) named the disease COVID-19. As of March 2, 2020, the number of confirmed cases in China had exceeded 80,000 and the number of cases outside China had exceeded 10,000, resulting in more than 3,000 deaths and a crude case fatality rate close to $3 \%(1)$, posing a huge threat to human life and safety. Up to date, the number of COVID-19 patients is still rapidly increasing worldwide, which is a threat to the health and lives of people all over the world. The main clinical symptoms of COVID-19 are fever, dry cough, and fatigue; while the main abnormalities in laboratory parameters are lymphopenia, elevated liver enzymes, elevated LDH, CRP, and erythrocyte sedimentation rate (1-5). Elevated troponin and D-dimer are observed in severe cases (6). In previous studies, 173 of 1,099 patients were clinically classified as severe or critically ill, with a severity rate of $15.7 \%$ (3). Different studies reported mortality rates varied $4.3 \%$ to $14.6 \%$. Organ insufficiency and coagulopathy were closely associated with high mortality $(7,8)$.

There have been few reports on the analysis of coagulopathy in patients with COVID-19. A previous study reported abnormalities in coagulation-related parameters in 183 patients at Wuhan Tongji Hospital at admission and during hospitalization. The study found that prothrombin time, fibrinogen, D-dimer, and fibrinogen degradation products of patients in the nonsurviving group were elevated compared to those in the surviving group, suggesting that coagulopathy may be associated with prognosis, and may guide clinical treatment (6). To further investigate the correlation between coagulopathy and disease, we analyzed 303 patients in Shanghai diagnosed with novel coronavirus 
pneumonia and followed up on the dynamic changes of coagulation function in 240 patients who achieved clinical cure and were discharged.

\section{Subjects and Methods}

\subsection{Research subjects}

Between January 20 and February 24, 2020, 324 adult patients with confirmed COVID-19 were admitted to the Shanghai Public Health Clinical Center, of which a total of 303 patients were included in the study. Among them, there was 1 mild case, 276 moderate cases, 10 severe cases, and 16 critical cases. All patients were diagnosed and classified based on the criteria in the "Diagnosis and Treatment Protocols for Novel Coronavirus Pneumonia (7th draft edition)" (9). As of February 24, 2020, 240 patients had been discharged, including 1 mild case, 234 moderate cases, and 5 severe cases.

\subsection{Methods}

Clinical data and baseline coagulation function data was collected from the 303 patients at the time of admission. Among them, the coagulation function of the 240 patients, who recovered and were discharged, were retrospectively analyzed. These cases were divided into two groups based on disease severity: mild (including mild and moderate) and severe (including severe and critical). The baseline coagulation functions of the two groups were compared to assess the correlation between coagulation function and disease severity. The coagulation function of the 240 discharged patients at admission and at discharge were compared, and the changes in coagulation function were followed up.

\subsection{Statistics}

Data were processed using SPSS 19.0 statistical software. Non-normally distributed measurement data were presented using the median (interquartile range), and the Mann-Whitney test was used to compare between groups (for non-normally distributed data). Qualitative data were expressed as frequencies or rates, and the chi-square test or Fisher's exact test was used for comparisons between groups. Results with $p<0.05$ were considered statistically significant.

\section{Results}

\subsection{Baseline characteristics of patients with COVID-19}

A total of 303 patients diagnosed with COVID-19 were included in this study, consisting of 1 mild case, 276 moderate cases, 10 severe cases, and 16 critical cases. These cases were divided into two groups based on the disease severity: a mild group (including mild and moderate) and a severe group (including severe and critical); the rate of severe cases was $8.6 \%$. There were 158 males and 145 females, the median age was 51 years (Age range: 16-88 years). The median time from the disease onset to admission was 4 days (2-8 days). Mild and severe groups showed statistically significant differences in sex ratio, age distribution, and presence of chronic underlying diseases. In the severe group, there were more males, many patients were middle-aged or elderly, and there was a higher proportion of chronic underlying diseases, including 11 cases of hypertension, 8 cases of fatty liver disease, 5 cases of coronary heart disease, 6 cases of diabetes, 3 cases of chronic bronchitis, 1 case of malignant cancer, 1 case of chronic kidney disease, 1 case of cerebral infarction, and 1 case of hypothyroidism. There were no statistically significant differences between the two groups in smoking history or time from onset to admission (Table 1).

3.2. Analysis of abnormal coagulation parameters at admission

Coagulation function examination of the 303 patients at admission revealed abnormal parameters in a total of 209 $(69.0 \%)$ cases. The most common abnormal parameters were fibrinogen (FIB) (195 cases, 64.3\%) followed by D-dimer (129 cases, 42.6\%), prolonged prothrombin time (PT) (56 cases, 18.5\%), abnormal activated partial thromboplastin time (APTT) (66 cases, 21.8\%), which was increased in 57 cases $(18.8 \%)$ and decreased in 9 cases $(3.0 \%)$; and elevated fibrinogen degradation products (FDP) in 19 cases $(6.3 \%)$. The proportion of abnormal coagulation parameters in the severe group was higher than in the mild group (100\% vs. 66.1\%), of which the proportions of abnormal fibrinogen $(80.8 \%$ vs. $62.8 \%$ ), D-dimer ( $80.8 \%$ vs. $39.0 \%)$, activated partial thromboplastin time (34.6\% vs. $20.6 \%)$, prothrombin time $(38.5 \%$ vs. $16.6 \%)$, and fibrinogen degradation products $(19.2 \%$ vs. $5.1 \%)$ were higher than in the mild

Table 1. Comparison of baseline demographic characteristics of patients in the mild and severe groups

\begin{tabular}{|c|c|c|c|c|}
\hline Items & Mild $(n=277)$ & Severe $(n=26)$ & Statistic & $p$-value \\
\hline Males (patients (\%)) & $138(49.8 \%)$ & $20(76.9 \%)$ & $\mathrm{X}^{2}=7.0$ & 0.008 \\
\hline Age (years) & $50(36-63)$ & $65(63-76)$ & $Z=-4.736$ & $<0.001$ \\
\hline Chronic underlying disease (patients $(\%)$ ) & $100(36.1 \%)$ & $19(73.1 \%)$ & $X^{2}=13.6$ & $<0.001$ \\
\hline History of smoking (patients (\%)) & $12(4.3 \%)$ & $2(7.7 \%)$ & l & 0.607 \\
\hline Time from onset to admission (days) & $4.0(2.0-8.0)$ & $5.5(3.0-7.3)$ & $Z=-1.11$ & 0.268 \\
\hline
\end{tabular}


group (Figure 1).

Comparison of coagulation function parameters at admission between the mild and severe groups showed that median INR, PT, APTT, FIB, FDP, and D-dimer were higher in the severe group compared to the mild group (Table 2); all differences were statistically significant (Table 3 ).

Further analysis of fibrinogen and D-dimer, the two most frequently abnormal coagulation function parameters, showed that about half of the patients in the mild and severe groups had a mild elevation in fibrinogen, and the proportion of patients with fibrinogen $>7.0 \mathrm{~g} / \mathrm{L}$ in the severe group was significantly higher than in mild group $(19.1 \%$ vs. $5.7 \%)$. The proportion of patients with elevated D-dimer $<2$ ULN was $64.8 \%$ in the mild group but only $33.3 \%$ in the severe group. Further, in the severe group, more than $50 \%$ of patients had elevated D-dimer $>2$ ULN; particularly, the proportion with D-dimer $>10 \mathrm{ULN}$ was significantly higher in severe groups than in the mild group $(19.0 \%$ vs. $3.7 \%$ ) (Figure 2).
3.3. Characteristics in coagulation function at admission and at discharge

The 240 patients who were discharged included 1 mild case, 234 moderate cases, and 5 severe cases. The median time of hospital stay was 14 days (11-19 days). Comparing the coagulation function of patients at admission and at discharge showed that fibrinogen degradation products were elevated. Median INR, prothrombin time, and D-dimer at discharge were decreased compared to the values at admission, but the differences were not statistically significant. Median fibrinogen decreased and median APTT was significantly shortened (Table 4).

\section{Discussion}

We analyzed the baseline data of 303 patients collected at the time of admission and found a total of 26 severe and critical cases of COVID-19 diagnosed in the Shanghai area. The rate of occurrence of severe

\section{A}

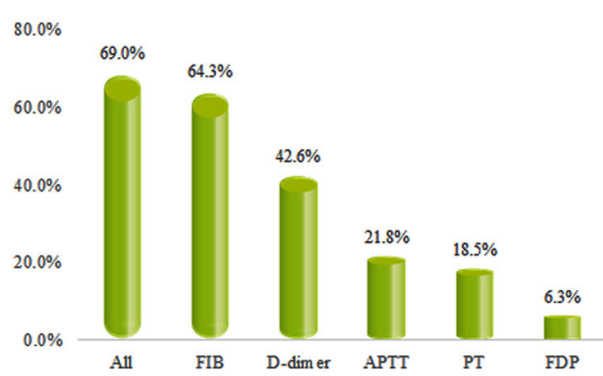

B

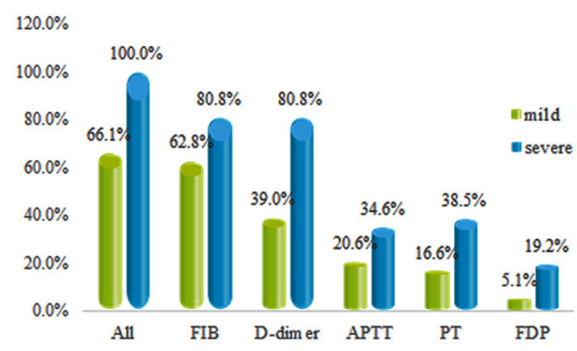

Figure 1. Proportion of abnormal coagulation function parameters at admission in the 303 patients in the mild and severe groups. A: Proportion of abnormal coagulation parameters at admission in the 303 patients. B: Proportion of abnormal coagulation parameters at admission in the mild and severe groups. Legend: green, mild; blue, severe.

Table 2. Coagulation function parameters at admission in the 303 patients

\begin{tabular}{|c|c|c|c|c|c|}
\hline Items & Reference range & Median & Interquartile range & Maximum & Minimum \\
\hline INR & & 1.01 & $0.97-1.05$ & 2.15 & 0.84 \\
\hline PT (s) & $11.0-14.0$ & 13.4 & $13.0-13.8$ & 24.4 & 11.6 \\
\hline APTT (s) & $32.0-43.0$ & 39.6 & $36.4-42.7$ & 110.6 & 28.5 \\
\hline FIB (g/L) & $2.0-4.0$ & 4.4 & $3.65-5.41$ & 10.5 & 1.34 \\
\hline $\mathrm{FDP}(\mu \mathrm{g} / \mathrm{mL})$ & $0-5.0$ & 1.05 & $0.58-2.09$ & 150 & 0.01 \\
\hline D-dimer $(\mu \mathrm{g} / \mathrm{mL})$ & $0-0.5$ & 0.45 & $0.31-0.81$ & 20.1 & 0.04 \\
\hline
\end{tabular}

The table shows the reference range, median, interquartile range, maximum, and minimum values of coagulation function parameters for the 303 admitted patients.

Table 3. Comparison of coagulation function parameters between the mild and severe groups

\begin{tabular}{|c|c|c|c|c|}
\hline Items & Mild $(n=277)$ & Severe $(n=26)$ & Statistic & $p$-value \\
\hline INR & $1.01(0.97-1.05)$ & $1.04(1.01-1.14)$ & $Z=-2.965$ & 0.003 \\
\hline PT (s) & $13.4(13.0-13.8)$ & $13.8(13.4-14.8)$ & $Z=-2.943$ & 0.003 \\
\hline APTT (s) & $39.2(36.3-42.4)$ & $43.2(41.0-49.7)$ & $Z=-3.792$ & $<0.001$ \\
\hline FIB (g/L) & $4.33(3.57-5.37)$ & $4.74(4.21-5.84)$ & $Z=-2.080$ & 0.038 \\
\hline $\operatorname{FDP}(\mu \mathrm{g} / \mathrm{mL})$ & $0.99(0.52-1.98)$ & $2.61(1.44-4.48)$ & $Z=-4.478$ & $<0.001$ \\
\hline D-dimer $(\mu \mathrm{g} / \mathrm{mL})$ & $0.43(0.31-0.77)$ & $1.04(0.73-1.72)$ & $Z=-5.156$ & $<0.001$ \\
\hline
\end{tabular}




\section{A}

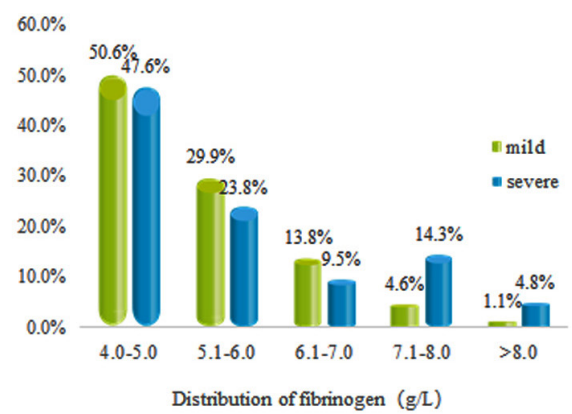

B

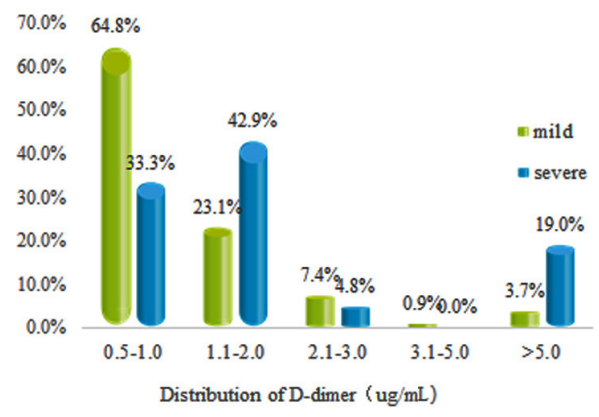

Figure 2. Distribution of fibrinogen and D-dimer values in the mild and severe groups. A: Distribution of fibrinogen (g/L), B: Distribution of D-dimer $(\mu \mathrm{g} / \mathrm{mL})$. Legend: green, mild; blue, severe.

Table 4. Comparison of coagulation function parameters in the 240 patients at admission and at discharge

\begin{tabular}{lcccr}
\hline Items & Admission & Discharge & Statistic & $p$-value \\
\hline INR & $1.01(0.97-1.05)$ & $1.00(0.96-1.03)$ & $\mathrm{Z}=-1.495$ & 0.135 \\
PT $(\mathrm{s})$ & $13.4(13.0-13.8)$ & $13.3(12.9-13.7)$ & $\mathrm{Z}=-1.477$ & 0.140 \\
APTT $(\mathrm{s})$ & $39.3(36.4-42.2)$ & $37.0(34.7-39.5)$ & $\mathrm{Z}=-5.895$ & $<0.001$ \\
FIB $(\mathrm{g} / \mathrm{L})$ & $4.42(3.61-5.24)$ & $3.82(3.27-4.79)$ & $\mathrm{Z}=-4.534$ & $<0.001$ \\
FDP $(\mu \mathrm{g} / \mathrm{mL})$ & $0.99(0.52-1.93)$ & $1.10(0.46-1.90)$ & $\mathrm{Z}=-0.058$ & 0.954 \\
D-dimer $(\mu \mathrm{g} / \mathrm{mL})$ & $0.44(0.31-0.78)$ & $0.43(0.29-0.71)$ & $\mathrm{Z}=-0.717$ & 0.473 \\
\hline
\end{tabular}

cases was $8.6 \%$, which was lower than previous reports $(3,7,8)$. The severe cases had a relatively high proportion of males, were relatively older, and had more underlying diseases, which is consistent with previous reports $(1,3)$. Analysis of coagulation function data showed that the incidence of abnormalities in conventional coagulation function parameters was higher in patients with severe cases compared to mild cases, and the magnitude of these increases was significant, suggesting that coagulopathy is more serious in severe patients, and significant coagulopathy correlates with the degree of disease severity to some extent (ด).

The incidence of coagulopathy in all patients at the time of admission showed abnormalities of varying degrees in conventional coagulation function parameters. In particular, the incidence of abnormal fibrinogen reached $64.3 \%$ and the occurrence of abnormal D-dimer was $42.6 \%$. These results suggest that fibrinogen and D-dimer are not only significantly increased in patients with severe cases but also increased to varying extent in a considerable proportion of patients with mild cases; however, a more significant increase was observed in patients with severe cases. This finding is consistent with previous reports (6). More significantly, we found that fibrinogen indicators improved significantly by the time of discharge, suggesting that fibrinogen levels normalized during recovery. This indicator may be used as a serum biomarker for predicting disease outcomes and good prognosis of COVID-19. Fibrinogen is a coagulation protein synthesized by the liver and promotes platelet aggregation, red blood cell adhesion and thrombosis, and is an important factor in coagulation and thrombosis. Based on autopsy and histopathological biopsy observations of patients with COVID-1, fibrinous exudates, intravascular hyaline thrombi, and pulmonary interstitial fibrosis were found in the alveoli, and microthrombi were found in the liver and kidney (9). Previous studies have reported that fibrin was abnormally elevated in the lungs of patients infected with SARS$\mathrm{CoV}$, and continuous excessive fibrin accumulation in the alveoli led to acute inflammation and chronic pulmonary fibrosis. Fibrin accumulation is a hallmark of acute respiratory distress syndrome and a reduced capacity to remove fibrin deposits results in poor clinical patient outcomes (10). Current research shows that SARS-CoV-2 is over $85 \%$ homologous to bat SARS-like coronavirus. So, it is speculated that a certain proportion of patients, especially critically ill patients, may have elevated fibrinogen after SARS-CoV-2 infection. Whether or not this is the underlying mechanism of the injury to the lung or other organs requires further study.

A recent retrospective analysis of 21 COVID19-related fatalities showed that the incidence of disseminated intravascular coagulation (DIC) was $71.8 \%$ (6), which warns us that we must be highly vigilant about the possibility of DIC in critically ill patients. Current research suggests that cytokine release syndrome (CRS) caused by immune imbalance following SARS-CoV-2 infection may be an important cause of diffuse microvascular injury. Researchers analyzed 30 immunological parameters in the blood of 33 COVID-19 patients and speculated that the mechanism of inflammatory damage may be $\mathrm{T}$ cell activation following SARS-CoV-2 infection and production of a large amount of granulocyte-macrophage colony-stimulating factor 
and IL-6, which induces a cascade of inflammatory factors (11). IL-6 can cause coagulopathies through many pathways. Cytokine storm interacts with coagulopathies to form a vicious cycle, which is directly correlated with poor prognosis (12). For patients with elevated IL-6, timely administration of the IL-6 inhibitor tocilizumab may improve CRS and reduce the risk of DIC (13). We observed a significant difference in D-dimer and FDP between the severe and mild groups. In particular, the patients in the high D-dimer interval, there was a significantly higher proportion of patients with severe cases than patients with mild cases, which may be related to the higher probability of DIC in critically ill patients. Therefore, these are important evaluation parameters for DIC. In addition to CRS caused by inflammatory factor storms in critically ill patients, septic shock occurs in a significant proportion of the population, which is also one of the common causes of DIC $(14,15)$.

There are also many limitations in this study. The research data comes from a single-center clinical retrospective study. The rate of exacerbation and the incidence of coagulopathies in patients may not be representative of the entire disease population, and there was no in-depth dynamic follow-up and analysis of the relationship between coagulation function and prognosis in critically ill patients. A multi-center study with a larger sample size is needed to verify our results. Nevertheless, our study suggests that coagulopathy is common among COVID-19 patients and that DIC-related parameters are significantly elevated in patients with severe cases compared to those with mild cases. Nearly $65 \%$ of patients had elevated fibrinogen to varying degrees, and the increase was more pronounced in critically ill patients. This indicator improved correspondingly as they recovered from the disease, suggesting that fibrinogen may be associated with the disease process.

\section{Acknowledgements}

This study was supported by a hospital-level project by Shanghai Public Health Clinical Center (KYGW-2018-18) and the National Science and Technology Major Project of China (2018ZX10302206).

\section{References}

1. Sohrabi C, Alsafi Z, O'Neill N, Khan M, Kerwan A, AlJabir A, Iosifidis C, Agha R. World Health Organization declares Global Emergency: a review of the 2019 Novel Coronavirus (COVID-19). Int J Surg. 2020. 2020 Apr;76:71-76.

2. Chen J, Qi T, Liu L, et al. Clinical progression of patients with COVID-19 in Shanghai, China. J Infect. 2020; 80:e1e6.

3. Guan WJ, Ni ZY, Hu Y, et al. Clinical Characteristics of Coronavirus Disease 2019 in China. N Engl J Med. 2020; doi: 10.1056/NEJMoa2002032.

4. Yao N, Wang SN, Lian JQ, Sun YT, Zhang GF, Kang WZ, Kang W. Clinical characteristics and influencing factors of patients with novel coronavirus pneumonia combined with liver injury in Shaanxi region. Zhonghua Gan Zang Bing Za Zhi. 2020; 28:E003. (in Chinese)

5. Zhang JJ, Dong X, Cao YY, Yuan YD, Yang YB, Yan YQ, Akdis CA, Gao YD. Clinical characteristics of 140 patients infected with SARS-CoV-2 in Wuhan, China. Allergy. 2020; doi: 10.1111/all.14238.

6. Tang N, Li D, Wang X, Sun Z. Abnormal Coagulation parameters are associated with poor prognosis in patients with novel coronavirus pneumonia. J Thromb Haemost. 2020; 18:844-847.

7. Chen N, Zhou M, Dong X, Qu J, Gong F, Han Y, Qiu Y, Wang J, Liu Y, Wei Y, Xia J, Yu T, Zhang X, Zhang L. Epidemiological and clinical characteristics of 99 cases of 2019 novel coronavirus pneumonia in Wuhan, China: a descriptive study. Lancet. 2020; 395:507-513.

8. Wang D, Hu B, Hu C, Zhu F, Liu X, Zhang J, Wang B, Xiang H, Cheng Z, Xiong Y, Zhao Y, Li Y, Wang X, Peng Z. Clinical Characteristics of 138 Hospitalized Patients With 2019 Novel Coronavirus-Infected Pneumonia in Wuhan, China. JAMA. 2020; doi: 10.1001/jama.2020.1585.

9. Diagnosis and Treatment Protocol for Novel Coronavirus Pneumonia 2020; Trial Version 7, Revised. http://www. kankyokansen.org/uploads/uploads/files/jsipc/protocol_ V7.pdf (accessed April 12, 2020).

10. Gralinski LE, Bankhead A, 3rd, Jeng S, et al. Mechanisms of severe acute respiratory syndrome coronavirus-induced acute lung injury. mBio. 2013 6; 4:pii: e00271-13.

11. Zhou Y, Fu B, Zheng X, Wang D, Zhao C, qi Y, Sun R, Tian Z, Xu X, Wei H. Aberrant pathogenic GM-CSF+ $\mathrm{T}$ cells and inflammatory CD14+CD16+ monocytes in severe pulmonary syndrome patients of a new coronavirus. bioRxiv. 2020; https://doi.org/10.1101/2020.02.12.945576.

12. Tanaka T, Narazaki M, Kishimoto T. Immunotherapeutic implications of IL-6 blockade for cytokine storm. Immunotherapy. 2016; 8:959-970.

13. Jiang H, Liu L, Guo T, Wu Y, Ai L, Deng J, Dong J, Mei $\mathrm{H}, \mathrm{Hu}$ Y. Improving the safety of CAR-T cell therapy by controlling CRS-related coagulopathy. Ann Hematol. 2019; 98:1721-1732.

14. Kitchens CS. Thrombocytopenia and thrombosis in disseminated intravascular coagulation (DIC). Hematology Am Soc Hematol Educ Program. 2009; 240-246.

15. Iba T, Levi M, Levy JH. Sepsis-Induced Coagulopathy and Disseminated Intravascular Coagulation. Semin Thromb Hemost. 2020; 46:89-95.

Received April 13, 2020; Revised April 22, 2020; Accepted April 23, 2020

${ }^{\S}$ These authors contributed equally to this work

*Address correspondence to:

Zhiping Qian, Department of Severe hepatopathy, Shanghai Public Health Clinical Center, Fudan University. No. 2901, Caolang Road, Jinshan District, 201508, Shanghai, China.

E-mail: qianzhiping@shphc.org.cn

Hongzhou Lu, Department of Infectious Disease and Immunology, Shanghai Public Health Clinical Center, Fudan University. No. 2901, Caolang Road, Jinshan District, 201508, Shanghai, China.

E-mail: luhongzhou@fudan.edu.cn

Released online in J-STAGE as advance publication April 30, 2020. 